\title{
On the Extraction of Time Information from Energy-Resolved Experiments
}

\author{
JEAN DURUP \\ Laboratoire des Collisions Atomiques et Moléculaires, † Bâtiment 351, Université \\ de Paris-Sud, 91405 ORSAY, France
}

It is well known that in principle energy-resolved and time-resolved experiments provide complementary information. A few examples are reviewed where the time evolution of rapidly relaxing molecular systems is or could be better studied by energy-resolved experiments: (1) the evolution of a pair of resonances coupled with each other through a set of continua, (2) an irreversible relaxation of the intermediate state in the two-photon excitation of a polyatomic molecule, and (3) the derivation of a "dissociation time" from the angular distribution of fragments from photodissociation by linearly polarized light. In the first case it is shown how all parameters necessary for a time-dependent description may be obtained from the measurement, as functions of the excitation wavelength, of both the total absorption cross section across the resonances and the partial cross section for a single exit channel. In the last two cases the relevance of a description in terms of an "eigentime" of the molecule is demonstrated.

\section{INTRODUCTION}

The main processes which take place in excited state evolution are (1) quasi-periodic motion of the initial wave packet along some coordinate (with possible loss of amplitude and/or phase), (2) decay into continuum states, and (3) interference between wave packets initially created in a coherent way.

In principle, any of these processes may be perfectly studied either in a time-resolved experiment where the relevant states are created

\footnotetext{
† Laboratoire associé au Centre National de la Recherche Scientifique.
} 
by a light pulse much shorter than any characteristic time of evolution of the system, or in an energy-resolved experiment where the energy resolution is much narrower than any state width and any spacing between neighboring stationary states.

In simple cases where a system consists of two closely-spaced discrete states, or of a discrete state coupled with a single continuum, it is easy to compare the resolution attained for the same observable (e.g., the spacing between the two states or the quantum beat period in the first case, the width of the discrete state or the decay rate in the second case) in either a time-resolved experiment with resolution $\delta \tau$ or an energy-resolved experiment with resolution $\delta E$. If we assume that for a visible-light excitation the best available $\delta \tau$ is one picosecond and the best available $\delta E$ corresponds to $\delta \nu=100 \mathrm{kHz}$, then it turns out that the time-resolved experiment will be the more accurate if the characteristic time to be measured is larger than $2 \pi^{-1 / 2}(\delta \tau / \delta \nu)^{1 / 2}$, i.e., here $1 \mathrm{~ns}$, and vice versa. For instance if $\tau=10 \mathrm{ps}$ the timeresolved experiment will have a resolution of $10^{-1}$ whereas the energyresolved experiment will have a resolution of $10^{-5}$. Thus, in contrast with intuition, ultra-fast relaxation phenomena should rather be studied using $\mathrm{cw}$, single-mode lasers.

However, we don't want only to record observations and be able to make correct predictions, but we also look for an understanding of the processes, and the information on time evolution generally is more illuminating than any other kind of information. Obviously too, in simple cases such as the above examples the output of the energyresolved experiments is easily translated into time information, but this is no more true for more complicated systems.

Thus the questions we are addressing in the present paper are how to design and interpret single-mode laser experiments on ultrafast relaxation phenomena, and in particular how to derive time information out of them. A recent example of such studies is the treatment by Lorquet $e t a l .{ }^{1}$ of the vibronic relaxation of molecular ions using the data from wavelength-dependent photoelectron spectroscopy.

In the present work we shall inspect in turn three other kinds of processes: (1) evolution of a pair of resonances coupled via a set of continua, (2) excitation of a polyatomic molecule by successive absorption of two photons, and (3) molecular photodissociation. 


\section{THE PROBLEM OF TWO DISCRETE STATES COUPLED VIA A SET OF CONTINUA}

We consider a pair of discrete states 1 and 2 embedded in an arbitrarily large number of degenerate continua, and we assume there is no other discrete state interacting with 1 and 2 . The problem is whether or not 1 and 2 are coupled with each other via the continua, that is to say whether or not after an initial excitation of only one of these states (by an adequately chosen transition) a part of the wave packet will reach the other one through the continua. As we will show the answer may be given by a set of two experiments where the total absorption cross section into the pair of resonances on the one hand and any partial absorption cross section corresponding to a single exit channel on the other hand, are recorded as functions of the excitation wavelength from a given initial state.

The theoretical treatment ${ }^{2}$ is an extension of the Fano theory of resonances. ${ }^{3-6}$ Instead of the familiar formula ${ }^{4}$ for the total cross section

$$
\sigma=\sigma_{b}+\sigma_{a} \frac{(q+\varepsilon)^{2}}{1+\varepsilon^{2}}
$$

where $\sigma_{a}$ is the cross section for direct excitation of the interactive continuum, $\sigma_{b}$ the background excitation cross section, $q$ the profile parameter, and

$$
\varepsilon=\frac{E-E_{r}}{\Gamma / 2}
$$

where $E$ is the photon energy, $E_{r}$ the resonance energy and $\Gamma$ the resonance width, we obtain in the two-resonance case the following formula: ${ }^{2}$

$$
\begin{aligned}
\sigma= & \sigma_{b}\left[\sin ^{2} \alpha\left(\left(\varepsilon_{1} \varepsilon_{2}-\sin ^{2} \alpha\right)^{2}+\left(\varepsilon_{1}+\varepsilon_{2}\right)^{2}\right)\right]^{-1} \\
& \times\left\{\left[\varepsilon_{1}^{2} \varepsilon_{2}^{2}+2 q_{1} \sin ^{2} \alpha \varepsilon_{1} \varepsilon_{2}^{2}+\varepsilon_{1}^{2}+2 \cos ^{2} \alpha \varepsilon_{1} \varepsilon_{2}+\left(\cos ^{2} \alpha\right.\right.\right. \\
& \left.+q_{1}^{2} \sin ^{2} \alpha\right) \varepsilon_{2}^{2}+2 q_{1} \sin ^{2} \alpha \varepsilon_{1}+2 q_{1} \sin ^{2} \alpha \cos ^{2} \alpha \varepsilon_{2} \\
& \left.+q_{1}^{2} \sin ^{4} \alpha\right] \sigma_{1}-2 \cos \alpha\left[\varepsilon_{1}^{2} \varepsilon_{2}^{2}+\varepsilon_{1}^{2}+\left(1+\cos ^{2} \alpha\right.\right. \\
& \left.-q_{1} q_{2} \sin ^{2} \alpha\right) \varepsilon_{1} \varepsilon_{2}+\varepsilon_{2}^{2}+\left(q_{1}+q_{2}\right) \sin ^{2} \alpha\left(\varepsilon_{1}+\varepsilon_{2}\right)
\end{aligned}
$$




$$
\begin{aligned}
& \left.+q_{1} q_{2} \sin ^{4} \alpha\right] \sigma_{1}^{1 / 2} \sigma_{2}^{1 / 2}+\left[\varepsilon_{1}^{2} \varepsilon_{2}^{2}+2 q_{2} \sin ^{2} \alpha \varepsilon_{1}^{2} \varepsilon_{2}\right. \\
& +\left(\cos ^{2} \alpha+q_{2}^{2} \sin ^{2} \alpha\right) \varepsilon_{1}^{2}+2 \cos ^{2} \alpha \varepsilon_{1} \varepsilon_{2}+\varepsilon_{2}^{2}+2 q_{2} \\
& \left.\left.\times \sin ^{2} \alpha \cos ^{2} \alpha \varepsilon_{1}+2 q_{2} \sin ^{2} \alpha \varepsilon_{2}+q_{2}^{2} \sin ^{4} \alpha\right] \sigma_{2}\right\}
\end{aligned}
$$

where $\sigma_{1}, \sigma_{2}, q_{1}, q_{2}, \varepsilon_{1}, \varepsilon_{2}$ generalize ${ }^{5}$ for the two resonances 1 and 2 the usual Fano parameters $\sigma_{a}, q, \varepsilon, E_{r}, \Gamma$, with

$$
\varepsilon_{1}=\frac{E-E_{r 1}}{\Gamma_{1} / 2} \quad \varepsilon_{2}=\frac{E-E_{r 2}}{\Gamma_{2} / 2}
$$

The additional parameter $\alpha$ of eqn (3) is defined in the following way. To each of the resonances 1 and 2 there corresponds ${ }^{4}$ an "interactive continuum," $C_{1}$ and $C_{2}$ respectively, which is that particular linear combination of all degenerate continua which bears all the interaction with the resonance. Now

$$
\cos \alpha=\left\langle C_{1} \mid C_{2}\right\rangle
$$

Eq. (3) applies only when $\alpha \neq 0$; if $\alpha=0$ one finds instead Eq. (65) of Ref. 3.

It is easily shown that the interaction $V_{12}$ between resonances 1 and 2 through the set of continua is given by

$$
V_{12}=\frac{\left(\Gamma_{1} \Gamma_{2}\right)^{1 / 2}}{2 \pi} \cos \alpha
$$

Thus the time evolution of state 1 or 2 , if initially selectively excited by a short light pulse, can be inferred from the knowledge of the various parameters of Eqn. (3).

Figure 1 shows that in the case of the $\left(c^{4} \Sigma_{u}^{-}\left(3 s \sigma_{g}\right), v=0,1\right)$ Rydberg levels of $\mathrm{O}_{2}$ the total photoionization cross section ${ }^{7}$ may be fitted well to Eq. (3). However, Eq. (3) is a rational fraction in $E$ with 9 independent parameters whereas the total number of physically significant parameters is $10\left(\sigma_{1}, \sigma_{2}, q_{1}, q_{2}, E_{r 1}, E_{r 2}, \Gamma_{1}, \Gamma_{2}, \alpha, \sigma_{b}\right)$, so that the fitting in Figure 1 is indeterminate.

This indetermination reappears for each partial cross section. ${ }^{2}$ However if one measures the partial cross section for a single exit channel (continuum $C_{j}$ ), in addition to the total cross section, all physical parameters entering in both total and partial cross sections may be determined unambiguously. 


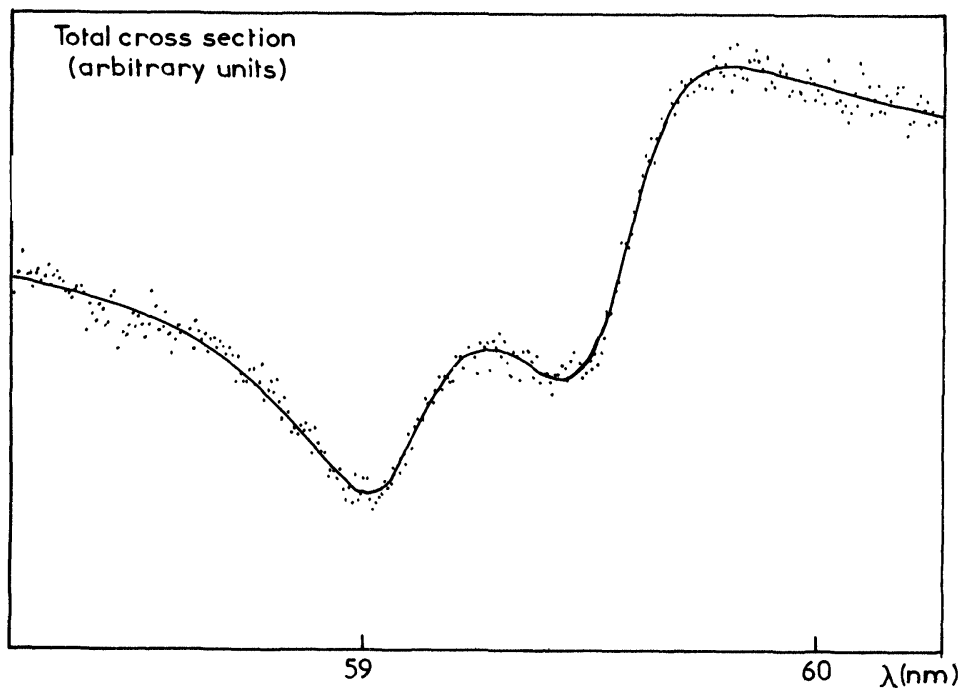

FIGURE 1 Fitting of $\mathrm{O}_{2}$ total photoionization cross section ${ }^{7}$ across a pair of resonances to Eq. (3).

The expression of the partial cross section in this particular case of a single exit channel reads: ${ }^{2}$

$$
\begin{aligned}
\sigma_{j}= & \sigma_{j a}+\left[\left(\varepsilon_{1} \varepsilon_{2}-\sin ^{2} \alpha\right)^{2}+\left(\varepsilon_{1}+\varepsilon_{2}\right)\right]^{-1} \\
& \times\left\{\left(1+q_{1}^{2}\right)\left[\left(r_{1}-\rho \cos \alpha r_{2}\right)^{2}+\varepsilon_{2}^{2} r_{1}^{2}\right]\right. \\
& +\left(1+q_{2}^{2}\right)\left[\left(r_{2}-\frac{\cos \alpha}{\rho} r_{1}\right)^{2}+\varepsilon_{1}^{2} r_{2}^{2}\right] \\
& +2\left[\left(1+q_{1} q_{2}\right)\left(\varepsilon_{1} \varepsilon_{2} r_{1} r_{2}+\left(r_{1}-\rho \cos \alpha r_{2}\right)\left(r_{2}-\frac{\cos \alpha}{\rho} r_{1}\right)\right)\right. \\
& \left.+\left(q_{1}-q_{2}\right)\left(\left(\varepsilon_{2}-\varepsilon_{1}\right) r_{1} r_{2}+\cos \alpha\left(\varepsilon_{1} \rho r_{2}^{2}-\frac{\varepsilon_{2}}{\rho} r_{1}^{2}\right)\right)\right] \\
& +2\left[\left(q_{1}\left(\varepsilon_{1} \varepsilon_{2}^{2}+\varepsilon_{1}+\varepsilon_{2} \cos \alpha\right)-\varepsilon_{2}^{2}-\sin ^{2} \alpha\right) r_{1}\right. \\
& -\left(\varepsilon_{1} \varepsilon_{2}-\sin ^{2} \alpha+q_{1}\left(\varepsilon_{1}+\varepsilon_{2}\right)\right) \rho \cos \alpha r_{2}
\end{aligned}
$$




$$
\left.\begin{array}{l}
+\left(q_{2}\left(\varepsilon_{1}^{2} \varepsilon_{2}+\varepsilon_{2}+\varepsilon_{1} \cos ^{2} \alpha\right)-\varepsilon_{1}^{2}-\sin ^{2} \alpha\right) r_{2} \\
\left.-\left(\varepsilon_{1} \varepsilon_{2}-\sin ^{2} \alpha+q_{2}\left(\varepsilon_{1}+\varepsilon_{2}\right)\right) \frac{\cos \alpha}{\rho} r_{1}\right] \sigma_{j a}^{1 / 2}
\end{array}\right\}
$$

where $\sigma_{j a}$ is the cross section for direct excitation of the $C_{j}$ continuum,

$$
\begin{gathered}
\rho=\left(\sigma_{1} / \sigma_{2}\right)^{1 / 2} \\
\left\{\begin{array}{l}
r_{1}= \pm\left(\sigma_{1} \Gamma_{j 1} / \Gamma_{1}\right)^{1 / 2} \\
r_{2}= \pm\left(\sigma_{2} \Gamma_{j 2} / \Gamma_{2}\right)^{1 / 2}
\end{array}\right.
\end{gathered}
$$

$\Gamma_{j 1}$ and $\Gamma_{j 2}$ being the partial widths of resonances 1 and 2 in the $j$ channel.

Thus now together expressions (3) and (7) depend on 13 independent mathematical parameters which are functions of 13 physical parameters (the former 10 ones plus $\sigma_{j a}, \Gamma_{j 1}$ and $\Gamma_{j 2}$ ) which now may be all determined.

It is easily seen that if the total and partial cross sections, $\sigma$ and $\sigma_{j}$, are not absolute ones, and even if they are not normalized to each other, all parameters except $\sigma_{1}, \sigma_{2}, \sigma_{j a}, \Gamma_{j 1}, \Gamma_{j 2}$ will still be unambiguously determined, whereas the five ones just quoted will be given in arbitrary units.

Such an analysis is in progress on the basis of the experimental data of Morin et $a l^{8}$ on the decay of the above-mentioned $\mathrm{O}_{2}$ resonance pair into selected levels of $\mathrm{O}_{2}^{+}\left(b^{4} \Sigma_{g}^{-}\right)$.

\section{ANGULAR EFFECTS IN THE TWO-PHOTON EXCITATION OF A POLYATOMIC MOLECULE}

The problem is to design an experiment for measuring under steady conditions, using cw lasers, a fast decay rate of a directly-excited quasi-continuum of states of a polyatomic molecule into a bath of even much more densely spaced states.

Let $\{j\}$ be the ensemble of states of interest decaying into the bath of states $\{r\}$. Let us assume that $\{j\}$, but not $\{r\}$, is optically connected with both a lower state $i$ and an upper state $f$. The experiment consists in exciting the molecule from $i$ to $f$ with two cw lasers of tunable frequencies $\nu_{1}$ and $\nu_{2}$ such that $h\left(\nu_{1}+\nu_{2}\right)$ is fixed to the $i \rightarrow f$ excitation energy, and that $h \nu_{1}$ brings the molecule into the region of states $\{j\}$ (see Figure 2). 


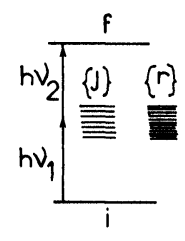

FIGURE 2 Scheme of the model experiment discussed in Section 3.

Under low-field conditions, the amount of state $f$ produced within a large time $t$ after the molecules entered the laser field is proportional to: ${ }^{9}$

$$
I \sim\left|\int_{0}^{t} d \theta \int_{0}^{t-\theta} \mathrm{d} \tau\left\langle f\left|T^{\prime}\right| \exp \left(-i \frac{H_{0}-E}{\hbar} \tau\right)|T| i\right\rangle\right|^{2}
$$

where $T$ and $T^{\prime}$ are the optical transition operators associated with both lasers, $H_{0}$ includes the molecular hamiltonian and the field hamiltonian (but not the molecule-field interaction), and $E$ is the energy of state $f$. Practically, $I$ may be monitored by the fluorescence of $f$.

$T$ and $T^{\prime}$ here depend on the angular coordinates of the molecule, if both lasers are polarized. Let $\alpha$ be the angle between the laser polarizations. Clearly the dependence of $I$ will reflect the time during which the molecule in states $\{j\}$ is allowed to rotate until being scavenged into states $\{r\}$. Thus rotation is an internal clock in such a steady experiment. The method obviously is of interest if, and only if, the $\{j\} \rightarrow\{r\}$ relaxation time is shorter than, or comparable to, the rotational period of the molecule, and therefore in the picosecond or subpicosecond range. As discussed in the Introduction it is just the range where energy-resolved experiments are of paramount interest.

The output of an experiment, such as described above, would be the anisotropy parameter of the $I(\alpha)$ distribution. It may be expressed $^{9}$ as an explicit function of the theoretical decay width $\Gamma$, which thus can be extracted from the experiment. In the case under consideration $\Gamma$ could not be directly measured as a width since it is larger than the rotational spacing.

On another hand, it turns out that when $t$ is large, which corresponds to the practical situation, expression (10) is equivalent to the simple 
expression:

$$
I \sim\left|\int_{0}^{\infty} d \tau\left\langle f\left|T^{\prime}\right| \exp \left(-i \frac{H_{0}-E}{\hbar} \tau\right)|T| i\right\rangle\right|^{2}
$$

providing the intermediate states $\{j\}$ (of which the $T|i\rangle$ ket is some linear combination) undergoes an irreversible decay process to some other states (here the $\{r\}$ set). In effect the second integral in (10) has a constant value as soon as $(t-\theta)$ is much larger than the decay time constant $(\hbar \mid \Gamma)$, so that at large $t$ the first integral only provides a constant factor $t$.

Expression (11) may be described in the following way. The molecule is excited from an initial state $i$ through a transition operator $T$; we define an eigentime $\tau$ of the molecule as being 0 at the time it gets excited, its wavefunction at that zero time being $T|i\rangle$; the molecule then evolves according to the evolution operator

$$
\exp \left(-i \frac{H_{0} \tau}{\hbar}\right)
$$

Whatever occurs thereafter to the molecule is subject only to the condition that any observable final state should obey energy conservation. This applies in particular to the final state $f \exp (-i(E / \hbar) \tau)$; hence the bracket in (11) represents the building-up of the $f$ component in the total wavefunction. Thus under low-field conditions (small $T^{\prime}$ ) and providing (12) includes an $\exp (-(\Gamma / \hbar) \tau)$ decay term, expression (11) gives the final weight of state $f$ in the squared total wavefunction and therefore the $f$ signal.

This "eigentime" description of the evolution of the excited molecule thus seems to be adequate for a comprehensive description of irreversible relaxation in an energy-resolved experiment. It would not be correct in a problem with reversible relaxation.

\section{THE ANGULAR DISTRIBUTION OF PHOTODISSOCIATION FRAGMENTS AND THE "DISSOCIATION TIME"}

It has always been considered intuitively that the angular distribution of fragments issuing from photodissociation by a linearly polarized light was dependent on the rotation which the molecule undergoes from the instant it absorbs the photon up to the instant the dissociation process is essentially accomplished. ${ }^{10}$ 
However, even from a classical viewpoint, this does not define a "dissociation time," which strictly speaking should be infinite. The intuitive definition of the dissociation time consists in extrapolating the initial motion of the excited molecule as though its geometry was not changing, as it does by the dissociation process, and to compute the time which then would be needed for the molecular axis to reach the orientation which will eventually be that of the fragment pair in reality, as experimentally observed. This definition ${ }^{9}$ actually is similar to the familiar definition of the lifetime in an exponential decay process, which coincides with the intersection of the time axis with the linear extrapolation at zero time of the decay law.

We shall now show how the formalism developed in Section 3 for an "eigentime" treatment of an energy-resolved experiment may be applied to this problem. The following description completes and makes more rigorous the one we gave in an earlier publication. ${ }^{9}$

We start from Eq. (11) as discussed in Section 3: if it provides the correct result for the observables it may be used for a description of the time evolution of the molecule.

We assume for the sake of simplicity that the molecule is linear and has zero $\Omega$ both in ground and excited states. The $|i\rangle$ state in Eq. (11) now is designated as $|i, v, J, M\rangle$; the $|f\rangle$ state is designated as $|j, E, \theta, \phi\rangle$ as observed experimentally by the energy and flight direction of the photofragment; there is no longer a $T^{\prime}$ operator but a simple projection of the intermediate state

$$
\left.\exp \left(-i \frac{H_{0}}{\hbar} \tau\right)|T| i\right\rangle
$$

on the final state

$$
\exp \left(-i \frac{E}{\hbar} \tau\right)|f\rangle
$$

Expansion of $T|i\rangle$ over the infinite set of rovibronic wavefunctions $\left|j, E^{\prime}, J^{\prime}, M\right\rangle$ of the vibrational continuum of the upper electronic state $j$, with $J^{\prime}=J \pm 1$, yields

$$
\begin{aligned}
|T| i\rangle=(J-M & +1) \int d E^{\prime} R\left(E^{\prime}, J+1\right)\left|j, E^{\prime}, J+1, M\right\rangle \\
& +(J+M) \int d E^{\prime} R\left(E^{\prime}, J-1\right)\left|j, E^{\prime}, J-1, M\right\rangle
\end{aligned}
$$


with

$$
R\left(E^{\prime}, J^{\prime}\right)=\left\langle E^{\prime}, J^{\prime}\left|m_{i j}\right| v, J\right\rangle
$$

where $m_{i j}$ is the vibrational-coordinate dependent electronic transition moment, and where the bracket notation means integration over vibration coordinates only. The angular part of expression (15) is just the expansion of $\cos \theta Y_{J M}(\theta, \phi)$ over $Y_{J+1, M}(\theta, \phi)$ and $Y_{J-1, M}(\theta, \phi)$.

Expression (13) then becomes:

$$
\begin{array}{r}
\int d E^{\prime} \exp \left(-i \frac{E^{\prime}}{\hbar} \tau\right)\left\{(J-M+1) R\left(E^{\prime}, J+1\right)\left|j, E^{\prime}, J+1, M\right\rangle\right. \\
\left.+(J+M) R\left(E^{\prime}, J-1\right)\left|j, E^{\prime}, J-1, M\right\rangle\right\}
\end{array}
$$

which inserted into Eq. (11), along with (14), yields

$$
\begin{aligned}
I \sim & \mid \int d E^{\prime} \int_{0}^{\infty} d \tau \exp \left(-i \frac{E^{\prime}-E}{\hbar} \tau\right)\left\{(J-M+1) R\left(E^{\prime}, J+1\right)\right. \\
& \times\left\langle j, E, \theta, \phi \mid j, E^{\prime}, J+1, M\right\rangle+(J+M) R\left(E^{\prime}, J-1\right) \\
& \left.\times\left\langle j, E, \theta, \phi \mid j, E^{\prime}, J-1, M\right\rangle\right\}\left.\right|^{2} \\
= & \mid(J-M+1) R(E, J+1)\langle E, \theta, \phi \mid E, J+1, M\rangle \\
& +\left.(J+M) R(E, J-1)\langle E, \theta, \phi \mid E, J-1, M\rangle\right|^{2}
\end{aligned}
$$

Expression (18) is the correct expression. ${ }^{11}$

We therefore may interpret expression (13) as described at the end of Section 3, as representing the evolution at time $\tau$ of the molecule excited at time 0 .

To do this we shall decompose the hamiltonian $H_{0}$ into:

$$
H_{0}=H_{\text {ev }}+H_{\text {rot }}
$$

where $H_{\mathrm{ev}}$ includes the vibronic part of the molecular hamiltonian and the hamiltonian of the field, and $H_{\text {rot }}$ is the rotational part of the molecular hamiltonian. On the other hand, instead of expanding $T|i\rangle$ as in (15) over rovibrational wavefunctions of state $j$ corresponding to $J^{\prime}=J \pm 1$, we separate it as

$$
T|i\rangle=\cos \theta T_{\text {ev }}|i, v, J, M\rangle
$$

where $T_{\mathrm{ev}}$ is the vibronic part of the dipole transition operator. 
Thus

$T|i\rangle \sim\{(J-M+1)|J+1, M\rangle+(J-M)|J-1, M\rangle\} T_{\text {ev }}|i, v ; J, M\rangle$

where $|i, v ; J, M\rangle$ stands for the vibronic part of $|i, v, j, M\rangle . H_{0}$, defined in (19), acting on (21) leads to

$$
\begin{aligned}
\left.H_{0}|T| i\right\rangle= & \left\{(J-M+1)|J+1, M\rangle\left[\frac{(J+1)(J+2) \hbar^{2}}{2 I}+H_{\mathrm{ev}}\right]\right. \\
& \left.+(J+M)|J-1, M\rangle\left[\frac{(J-1) J \hbar^{2}}{2 I}+H_{\mathrm{ev}}\right]\right\} T_{\mathrm{ev}}|i, v ; J, M\rangle
\end{aligned}
$$

where $I$ here stands for the moment of inertia of the molecule.

We now look for the angular part of (13) at small $\tau$. In first order with respect to $\tau$ we have no further action of $H_{\mathrm{ev}}$ on the terms with $I$, and therefore

$$
\begin{aligned}
\left.\exp \left(-i \frac{H_{0}}{\hbar} \tau\right)|T| i\right\rangle \sim & \left\{(J-M+1)|J+1, M\rangle \exp \left(-i \frac{(J+1)(J+2) \hbar}{2 I} \tau\right)\right. \\
& \left.+(J+M)|J-1, M\rangle \exp \left(-i \frac{(J-1) J \hbar}{2 I} \tau\right)\right\} \\
& \left.\times \exp \left(-i \frac{H_{\mathrm{ev}}}{\hbar} \tau\right)\left|T_{\mathrm{ev}}\right| i, v ; J, M\right\rangle
\end{aligned}
$$

We can now derive the instantaneous angular distribution $F(\theta, \phi, \tau)$ of the molecular axis as the norm of expression (23) integrated over the vibronic coordinates only. Due to the hermiticity of the evolution operator we get:

$$
F(\theta, \phi, \tau) \sim\left\langle i, v ; J, M\left|T_{\mathrm{ev}}^{*} A_{J M}(\theta, \phi, \tau) T_{\mathrm{ev}}\right| i, v ; J, M\right\rangle
$$

where

$$
\begin{aligned}
A_{J M}(\theta, \phi, \tau)= & (J-M+1)^{2}\left[P_{J+1}^{M}(\cos \theta)\right]^{2}+(J+M)^{2}\left[P_{J-1}^{M}(\cos \theta)\right]^{2} \\
& +2(J-M+1)(J+M) P_{J+1}^{M}(\cos \theta) P_{J-1}^{M} \\
& \times(\cos \theta) \cos \frac{(2 J+1) \hbar}{I} \tau
\end{aligned}
$$

Following now a purely algebraic development similar to that of Eqs. (42)-(45) in Ref. (9), we obtain in the lower order in $\tau$ an 
instantaneous angular distribution characterized by an anisotropy parameter (after averaging over all $\boldsymbol{M}$ ):

$$
\beta=2-\frac{12 J(J+1)}{\hbar^{2}}\left(B^{2}\right)_{\mathrm{av}} \tau^{2}
$$

where $\left(B^{2}\right)_{\mathrm{av}}$ is the expectation value of $\left(\hbar^{2} / 2 I\right)^{2}$ over the vibrational part of $T_{\mathrm{ev}}|i, v ; J, M\rangle$. The latter, of course, reduces to the vibrational wavefunction of the initial $|i, v, J, M\rangle$ state if the transition obeys the Franck-Condon approximation; otherwise it is the product of this vibrational wavefunction by the vibrational-coordinate dependent electronic transition moment.

Our final equation (26) thus provides the definition of the "dissociation time" we were looking for, which is obtained by equating the R.H.S. to the experimentally observed $\beta$.

\section{References}

1. A. J. Lorquet, J. C. Lorquet, J. Delwiche and M. J. Hubin-Franskin, J. Chem. Phys. 76, 4692 (1982).

2. J. Durup and M. Lavollée, 9è Coll. Phys. Coll. Atom. Electron. (Nice, France 1982), to be published.

3. U. Fano, Phys. Rev. 124, 1866 (1961).

4. U. Fano and J. W. Cooper, Phys. Rev. A 137, 1364 (1965).

5. F. H. Mies, Phys. Rev. 175, 164 (1968).

6. F. Combet-Farnoux, Phys. Rev. A 25, 287 (1982).

7. A. Tabché-Fouhailé, I. Nenner, P. M. Guyon and J. Delwiche, J. Chem. Phys. 75, 1129 (1981).

8. P. Morin, I. Nenner, P. M. Guyon, L. F. A. Ferreira and K. Ito, Chem. Phys. Lett. 92, 103 (1982); P. Morin, I. Nenner, M. Y. Adam, M. J. Hubin-Franskin, J. Delwiche, H. Lefebvre-Brion and A. Giusti-Suzor, Chem. Phys. Lett. 92, 609 (1982).

9. J. Durup, Comments Atom. Molec. Phys. 12, 255 (1983).

10. M. Dzvonik, S. Yang and R. Bersohn, J. Chem. Phys. 61, 4408 (1974).

11. R. N. Zare, Ph.D. Thesis, Harvard University, 1964; Molec. Photochem. 4, 1 (1972). 